\title{
A robust Bayesian methodology for damage localization in plate-like structures using ultrasonic guided-waves
}

\author{
Sergio Cantero-Chinchilla ${ }^{\mathrm{a}, \mathrm{d}, *}$, Juan Chiachío ${ }^{\mathrm{b}}$, Manuel Chiachío ${ }^{\mathrm{c}}$, Dimitrios Chronopoulos ${ }^{\mathrm{a}}$, Arthur Jones ${ }^{\mathrm{a}}$ \\ a Institute for Aerospace Technology \& The Composites Group, The University of Nottingham, NG7 2RD, United Kingdom \\ $b$ Department of Naval Architecture, Ocean \& Marine Engineering, University of Strathclyde, Glasgow, G4 OLZ, United Kingdom \\ c Dept. Structural Mechanics \& Hydraulics Engineering, University of Granada, 18001, Spain \\ d Aernnova Engineering Division S.A., Madrid, 28034, Spain
}

\begin{abstract}
SHM methods for damage detection and localization in plate-like structures have typically relied on signal postprocessing techniques applied to ultrasonic guided-waves. The time of flight is one of these signals features which has been extensively used by the SHM community for damage localization. One approach for obtaining the time of flight is by applying a particular time-frequency transform to capture the frequency and energy content of the wave at each instant of time. To this end, the selection of a suitable methodology for time-frequency transform among the many candidates available in the literature has typically relied on experience, or simply based on considerations about computational efficiency. In this paper, a full probabilistic method based on the Bayesian inverse problem is proposed to rigorously provide a robust estimate of the time of flight for each sensor independently. Then, the robust prediction is introduced as an input to the Bayesian inverse problem of damage localization. The results reveal that the proposed methodology is able to efficiently reconstruct the damage localization within a metallic plate without the need to assume a specific a priori time-frequency transform model.
\end{abstract}

Keywords: Bayesian inverse problem, Ultrasonic guided-waves, Time of flight, Damage localization, Multiple damage areas, Structural Health Monitoring

\section{1. Introduction}

Damage reconstruction and localization in plate-like structures using guided-waves based SHM have been mainly 3 addressed using post-processing techniques applied to ultrasonic signals [1]. The exploration of large areas with a

4 small attenuation [2] is one of the most remarkable characteristics that has led industries, such as the aerospace in5 dustry, to focus on guided-waves (e.g. the "PAMELA" system [3-5]). Other approaches that use acoustic-based SHM 6 methods to localize damage in thin-walled structures are also available nowadays. These can be broadly classified 7 into (1) passive sensing diagnostics (PSD) and (2) active sensing diagnostics techniques (ASD). In contrast to PSD 8 techniques, which are based on sensors in "listening-mode" (e.g., acoustic emission) [6-12], ASD techniques for

\footnotetext{
* Corresponding Author

Email address: Sergio. CanteroChinchilla1@nott ingham. ac. uk (Sergio Cantero-Chinchilla)
} 


\begin{tabular}{|c|c|c|c|}
\hline \multicolumn{4}{|c|}{ Nomenclature } \\
\hline$a, b$ & scaling and time-shift factors & \multirow{2}{*}{\multicolumn{2}{|c|}{$\mathrm{ToF}_{M}^{(a-s)}$ modeled ToF between actuator $a$ and sensor }} \\
\hline$c$ & normalizing constant in Bayes' Theorem & & \\
\hline$D^{(k)}$ & mean of the hyper-robust model for the $k$-th & \multicolumn{2}{|c|}{$\mathscr{U}(\cdot, \cdot)$ uniform distribution } \\
\hline & sensor & $v$ & mean of prior PDF of the velocity \\
\hline$\hat{\mathbf{d}}^{(k)}$ & set of $\hat{d}_{j}$ in the $k$-th sensor & \multirow{2}{*}{\multicolumn{2}{|c|}{$\begin{array}{l}V_{a-d}, V_{d-s} \text { wave propagation velocity in paths } a-d \\
\text { and } d-s \text {, respectively }\end{array}$}} \\
\hline$\hat{d}_{j}^{(k)}$ & first energy peak provided by the $j$-th TF & & \\
\hline$\tilde{d}^{(k)}$ & $\begin{array}{l}\text { model in the } k \text {-th sensor } \\
\text { first energy peak measured in the } k \text {-th sensor }\end{array}$ & $w_{j}^{(k)}$ & $\begin{array}{l}\text { weight of the } j \text {-th TF model class in the } k \text {-th } \\
\text { sensor used in the hyper-robust model }\end{array}$ \\
\hline D & set of $D^{(k)}$ values for all the sensors & $X(t)$ & time series of the signal \\
\hline $\mathscr{D}^{(k)}$ & signal acquired in the $k$-th sensor & $X_{a}, Y_{a}$ & actuator coordinates \\
\hline$e$ & model error of ellipse-based ToF model & $X_{d}, Y_{d}$ & damage position coordinates \\
\hline $\begin{array}{l}g_{j}(\cdot) \\
h(t)\end{array}$ & $j$-th TF model & $X_{s}, Y_{S}$ & sensor coordinates \\
\hline $\begin{array}{l}n(t) \\
\mathbf{m}\end{array}$ & model parameters of ellipse-based $\mathrm{ToF}$ & $\alpha(t)$ & magnitude of the analytic signal \\
\hline & model purate & $\gamma$ & threshold parameter for AIMS algorithm \\
\hline $\mathscr{M}_{j}^{(k)}$ & $j$-th TF model class for the $k$-th sensor & $\varepsilon$ & error term in TF model \\
\hline $\mathbf{M}$ & set of model classes $\mathscr{M}_{j}$ & $\zeta$ & number of state of the chain in $\mathrm{M}-\mathrm{H}$ \\
\hline$N$ & number of sensors & $\boldsymbol{\Theta}$ & set of possible values of the parameters in the \\
\hline$N_{m}$ & number of model classes & & BIP \\
\hline$N_{i}^{A}$ & number of samples in $i$-th annealing level & $\boldsymbol{\theta}$ & set of ToF model parameters including $\sigma_{e}$ \\
\hline $\mathscr{N}(\cdot, \cdot)$ & Gaussian distribution & $\rho$ & scaling factor in the prior PDF of $\sigma_{\varepsilon}$ \\
\hline$P(\cdot)$ & probability & $\sigma_{\varepsilon}$ & standard deviation of $\varepsilon$ \\
\hline$p(\cdot)$ & probability density & $\sigma_{e}$ & standard deviation of model error of ellipse- \\
\hline$q(\cdot)$ & $\begin{array}{l}\text { proposal PDF in MCMC sampling algo- } \\
\text { rithms }\end{array}$ & & based ToF model \\
\hline$r$ & acceptance rate for the M-H algorithm & $\sigma_{M}$ & MAP value of $\sigma_{\varepsilon}$ \\
\hline$T_{s}$ & $\begin{array}{l}\text { number of samples generated in } \mathrm{M}-\mathrm{H} \text { algo- } \\
\text { rithm }\end{array}$ & $\sigma_{v}$ & $\begin{array}{l}\text { standard deviation of prior PDF of the veloc- } \\
\text { ity }\end{array}$ \\
\hline $\mathrm{ToF}_{\mathscr{Q}}^{(a-4}$ & s) measured ToF between actuator $a$ and sen- & $\Psi(t)$ & analyzing wavelet \\
\hline & sor $s$ & $\omega_{j}(t)$ & instantaneous frequency \\
\hline
\end{tabular}

- plate-like structures emit ultrasonic waves that interact with the structure and are measured by sensors [13]. Sparse 10 or phased-array sensors' layouts are placed so that the structure is actively interrogated on demand, which confers higher accuracy and reliability [14]. Potential safety and economical implications in condition-based maintenance are extra-motivations for the use of this SHM technique.

Moreover, the need for autonomous techniques that provide accurate health state indicators is specially crucial for 14 aerospace structures, which are based on a considerable number of critical structural components requiring frequent 
inspection. Once a damaged area is detected, i.e. through analysis of damage tolerance exceedance, proper operational decisions can be taken. Two general approaches are typically adopted for damage detection: (1) model-based inverse problems, whereby detailed damage information (e.g. the severity of damage as residual strength) [15] can be obtained from the measured signal at a considerable computational cost; and (2) inverse problems based on post-processed signal features, whereby other relevant information, e.g. the damage position or the damage severity, can be obtained more efficiently. With regards to the second approach, several damage reconstruction techniques have been reported in the literature [2, 16, 18]. Among them, the time-of-flight (ToF) has been extensively used as a signal feature for its efficiency in obtaining information about material properties along with damage localization using post-processing scattered signals.

Time-frequency (TF) representation techniques have been intensively used for the extraction of ToF as a signal feature. By TF representation, a frequency domain spectrum can be obtained at each instant of time [19], however the results slightly differ from each other depending on the adoption of the various approaches available in the literature. Amongst them, the Hilbert-Huang transform (HHT), the continuous wavelet transform (CWT), the short-time Fourier transform (STFT) and the Wigner-Ville distribution (WVD) [19-22], are some of the most commonly used techniques in ultrasonic guided-waves based SHM applications [23-25]. Typically, the selection of one among the available options has been based on the modeler's experience or based on specific TF resolution characteristics. However, the selection of an unappropriated model may result in a biased damage identification [26, 27] due to the disparate model assumptions and hypotheses adopted for each of them. In other words, the choice of a particular TF approach instead of another one is subject to epistemic uncertainty (i.e., lack of knowledge). Moreover, ultrasound-based damage localization conveys other sources of uncertainty which are mostly related with the measurement system and physical properties of the material. They might produce unreliable damage predictions should these uncertainties are not properly considered and quantified within the calculations.

To partially address this modeling issue, a number of researchers have proposed the use of probability-based methods [28,-30]. Among them, the Bayesian inverse problem (BIP) applied to ultrasound based damage localization is getting increasing attention within the SHM community, although it is still in its early stage. In [24], the BIP was successfully proven in localizing damage areas in aluminum plates. More recently, a BIP methodology to account for the anisotropy in the group velocity was proposed in [23] for composite laminates. Notwithstanding, there is still an evident need for a rigorous treatment of the uncertainty in modeling the damage localization using ultrasonic guided-waves based methods, overall when multiple damage locations are expected.

This paper proposes a multi-level Bayesian framework to rigorously account for the overall uncertainty in application to the problem of ultrasound-based damage localization using Lamb waves. The main novelty of this paper is that it provides a unified methodology to rationally address the problem of damage identification using ultrasounds from probabilistic Bayesian principles: first, the problem of TF model selection is addressed for a given experimental configuration based on posterior probabilities that assess the relative degree of belief [31] of a particular model over a set of candidates; then, the problem of damage identification and localization is carried out using a BIP based on sig- 


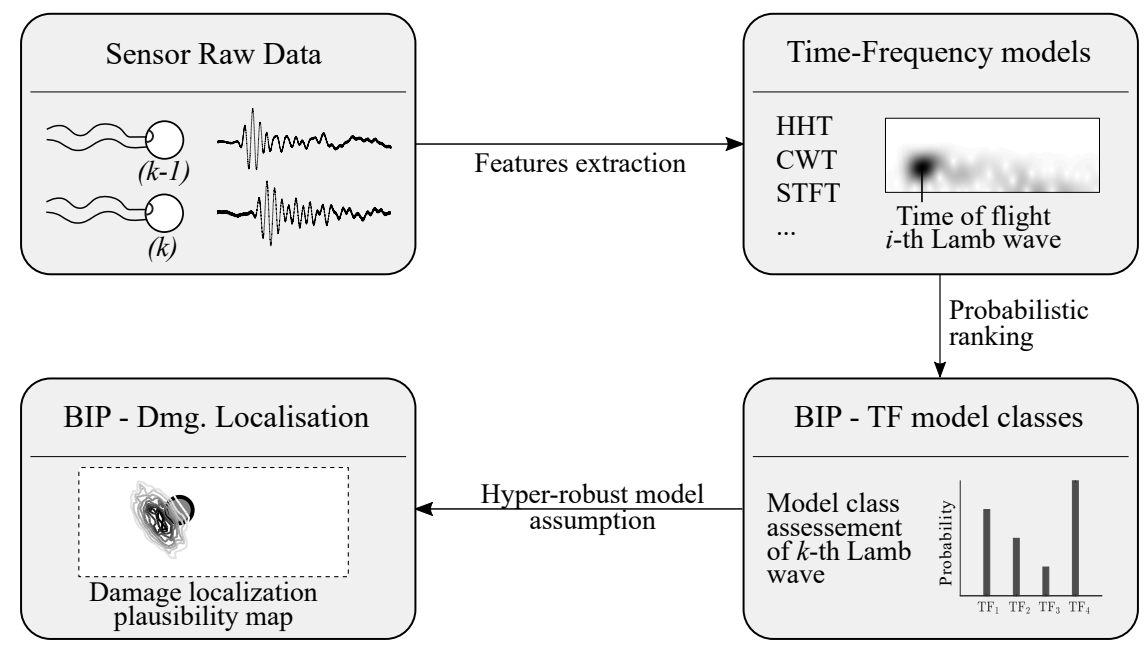

Figure 1: General workflow proposed to address the challenge of damage localization. Note that the signal data can be obtained by numerical (e.g. FEM) or experimental methods (e.g. using PZT transducers, a signal generator, and an oscilloscope) [13].

nal features adopting a hyper-robust TF model resulted from the first assessment level. To this end, once the raw data have been acquired, two BIPs are hierarchically formulated for each piezoelectric (PZT) sensor so that the outcome of the model selection problem is used as input for damage localization, as shown in Figure 1 . In this framework, uncertainties coming from (1) material's mechanical properties, (2) measurement errors, and (3) epistemic uncertainty in the TF model due to the Heisenberg principle [19, 30], are taken into account. The proposed approach relies on rigorous probability-logic assumptions for model class selection [32] and as such, it avoids experience-based decisions about the optimal post-processing technique. Here, probability is interpreted as a multi-valued logic that expresses the degree of belief of a proposition conditioned on the given information [32, 33]. The methodology is applied in two case studies using aluminum plates with one and two damaged areas, respectively. For the particular problem of damage localization, the asymptotic independent Markov sampling (AIMS) [34, 35] algorithm is adopted to solve the resulting Bayesian inverse problem, showing high efficiency in dealing with damage multi-modality. In general, the results show the efficiency of the proposed methodology in reconstructing the damage position in plate-like structures using guided-waves, while rigorously accounting for the modeling uncertainties in the reconstruction.

The remainder of the paper is organized as follows: Section 2 shows the TF models used in the proposed model selection problem. Section 3 comprises the probabilistic methodology used to obtain the robust estimate of the ToF for each sensor. The BIP principles used to obtain the damage localization are presented in Section 4. In Section 5 , the proposed framework is applied in two case studies to serve as example. Section 6 discusses the robustness of the proposed methodology. Finally, Section 7 provides concluding remarks. 


\section{Time-frequency models} window in the assessed signal [19, 37], as follows:

$$
g_{3}(\omega, t)=\frac{1}{2 \pi} \int_{-\infty}^{\infty} e^{-\mathrm{i} \omega \tau} X(\tau) h(\tau-t) \mathrm{d} \tau
$$

s6 where $X(t)$ is the time series, $h(t)$ is a window function, and $\omega$ denotes the frequency. The energy spectrum of an ${ }_{87}$ STFT is known as a spectrogram.

$$
g_{1}(t)=\sum_{j=1}^{n} \alpha_{j}(t) \exp \left(\mathrm{i} \int \omega_{j}(t) \mathrm{d} t\right)
$$

where $\alpha_{j}(t)$ is the magnitude of the analytic signal which is typically considered as the envelope of the input time series or directly the signal acquired by the sensor, $n$ is the number of IMF components, and $\omega_{j}(t)$ is the instantaneous frequency. Equation (1) represents the amplitude and instantaneous frequency as function of time.

\subsection{Continuous wavelet transform}

TF wavelets are used in the CWT to obtain the TF representation of the assessed signal, by:

$$
g_{2}(b, a)=\frac{1}{\sqrt{a}} \int_{-\infty}^{\infty} X(t) \overline{\Psi\left(\frac{t-b}{a}\right)} \mathrm{d} t
$$

where $X(t)$ represents the time series of the signal, $\Psi(t)$ denotes the analysing wavelet, $a>0$ is the scale factor, $b$ is the time-shift variable, and the overline denotes the complex conjugate [21, 36]. Remarkable time and frequency resolution are obtained using this model.

\subsection{Short-time Fourier transform}

Alternatively, the TF representation can be obtained with a STFT, which performs Fourier transforms to a moving$$
\text { STFT is known as a spectrogram. }
$$ 


\subsection{Wigner-Ville distribution}

The WVD can be interpreted as a measure of the signal's local time-frequency energy [37], and it is defined as follows:

$$
g_{4}(\omega, t)=\int_{-\infty}^{\infty} X\left(t+\frac{\tau}{2}\right) \overline{X\left(t-\frac{\tau}{2}\right)} e^{-i \omega \tau} \mathrm{d} \tau
$$

where $X(t)$ is the time series and the overline denotes the complex conjugate. This technique is highly effective in detecting and localizing Dirac impulses and sinusoids [19, 37].

\section{Bayesian model class ranking}

The TF models in Section 2 are just different alternatives based on a number of simplifying hypotheses and modeling assumptions to represent the same reality. Instead, for a particular model, the validity of such simplifying assumptions depends on the adopted values of certain model parameters (e.g. the dispersion parameter). Thus, to simultaneously identify both the plausibility of each TF model and the values of the model parameters that better suit the information coming from the raw ultrasonic data, a Bayesian inverse problem (BIP) is proposed here. Given a plate-like structure monitored through a set of PZT sensors, the BIP is addressed separately for each PZT sensor due to the potential differences between sensors, such as different working environments or manufacturing defects.

\subsection{Stochastic embedding of TF models}

Let us consider a candidate TF model defined by the relationship $g_{j}: \mathbb{R}^{n} \rightarrow \mathbb{R}$ between a discrete signal $\mathscr{D}^{(k)} \in \mathbb{R}^{n}$ acting as input and the model output $g_{j} \in \mathbb{R}$, where $k$ denotes the $k$-th sensor in the structure. Next, let $\hat{d}_{j}^{(k)} \in \mathbb{R}$ be the first energy peak observed in the scattered ultrasound signal, so that $\hat{d}_{j}^{(k)}=g_{j}\left(\mathscr{D}^{(k)}\right)$. Under the assumption that $g_{j}$ is only a candidate model over a set of alternatives [32] (e.g. like those described in Section 2), then the measured first peak, denoted here as $\tilde{d}^{(k)}$, would be more rigorously represented as an uncertain variable, as follows:

$$
\tilde{d}^{(k)}=g_{j}\left(\mathscr{D}^{(k)}\right)+\varepsilon
$$

where $\varepsilon$ is an uncertain error term which accounts for the discrepancy between $\hat{d}_{j}^{(k)}$ and $\tilde{d}^{(k)}$, namely the modeled and measured values for the first energy peak, respectively. Following the Principle of Maximum Information Entropy (PMIE) [32, 33], this error can be conservatively assumed to be modeled as a zero-mean Gaussian distribution with standard deviation $\sigma_{\varepsilon}$, i.e., $\varepsilon \sim \mathscr{N}\left(0, \sigma_{\varepsilon}\right)$. The PMIE enables a rational way to establish a probability model for the model error term such that it produces the largest uncertainty (largest Shannon entropy); the selection of any other probability model would lead to an unjustified reduction in such uncertainty [32]. Thus, following Equation (5), a 
probabilistic description of the TF model can be obtained as:

$$
p\left(\tilde{d}^{(k)} \mid \mathscr{M}_{j}^{(k)}, \sigma_{\varepsilon}\right)=\left(2 \pi \sigma_{\varepsilon}^{2}\right)^{-\frac{1}{2}} \exp \left(-\frac{1}{2}\left(\frac{\tilde{d}^{(k)}-g_{j}^{-1}\left(\mathscr{D}^{(k)}\right)}{\sigma_{\varepsilon}}\right)^{2}\right)
$$

where $\mathscr{M}_{j}^{(k)}$ denotes the $j$-th candidate model class within a set of $N_{m}$ available TF models $\mathbf{M}=\left\{\mathscr{M}_{1}^{(k)}, \ldots, \mathscr{M}_{j}^{(k)}, \ldots, \mathscr{M}_{N_{m}}^{(k)}\right\} . \quad$ Each model class is defined by the stochastic $\mathrm{TF}$ model given by Equation (6) along with the prior probability density function (PDF) of the model parameter $\sigma_{\varepsilon}, p\left(\sigma_{\varepsilon} \mid \mathscr{M}_{j}^{(k)}\right)$. This prior PDF represents the initial degree of belief of the values of $\sigma_{\varepsilon}$ within a set of possible values $\Theta \subseteq \mathbb{R}$ before the information from measurements is incorporated through Bayesian updating, as explained further below. For all the sensors in the structure, the stochastic model is defined independently, thus accounting for different potential sources of errors and uncertainties.

\subsection{Model parameters estimation}

Previously to obtain the model parameter updating from measurements, a preliminary assessment of the influence of the dispersion parameter $\sigma_{\varepsilon}$ in the model class assessment and ranking was carried out, which showed a relatively high sensitivity of the resulting model class assessment to the value of this parameter. Thus, a first stage of the BIP is conceived to obtain the set of most plausible values for $\sigma_{\varepsilon}$ given a set of data $\hat{\mathbf{d}}^{(k)}=\left\{\hat{d}_{1}^{(k)}, \ldots, \hat{d}_{N_{m}}^{(k)}\right\}$, which corresponds to a set of $N_{m}$ values obtained by adopting each TF model class. To this end, the posterior PDF $p\left(\sigma_{\varepsilon} \mid \hat{\mathbf{d}}^{(k)}, \mathscr{M}_{j}^{(k)}\right)$ of the dispersion parameter $\sigma_{\varepsilon}$ given the $j$-th TF model class $\left(\mathscr{M}_{j}^{(k)}\right)$, is required. Thus, by using Bayes' theorem, this posterior PDF is given by:

$$
p\left(\sigma_{\varepsilon} \mid \hat{\mathbf{d}}^{(k)}, \mathscr{M}_{j}^{(k)}\right)=c^{-1} p\left(\hat{\mathbf{d}}^{(k)} \mid \sigma_{\varepsilon}, \mathscr{M}_{j}^{(k)}\right) p\left(\sigma_{\varepsilon} \mid \mathscr{M}_{j}^{(k)}\right)
$$

where $c$ is a normalizing constant, so that:

$$
\int_{\Theta} p\left(\sigma_{\varepsilon} \mid \hat{\mathbf{d}}^{(k)}, \mathscr{M}_{j}^{(k)}\right) d \sigma_{\varepsilon}=\int_{\Theta} c^{-1} p\left(\hat{\mathbf{d}}^{(k)} \mid \sigma_{\varepsilon}, \mathscr{M}_{j}^{(k)}\right) p\left(\sigma_{\varepsilon} \mid \mathscr{M}_{j}^{(k)}\right) d \sigma_{\varepsilon}=1
$$

In Equation (7), $p\left(\hat{\mathbf{d}}^{(k)} \mid \sigma_{\varepsilon}, \mathscr{M}_{j}^{(k)}\right)$ is the likelihood function, which expresses how likely the data $\hat{\mathbf{d}}^{(k)}$ are reproduced by the stochastic model in Equation (6) if model class $\mathscr{M}_{j}^{(k)}$ is adopted, as shown in Figure 2. This likelihood function can be obtained by substitution of the values of $\hat{\mathbf{d}}^{(k)}$ as the output of the stochastic model, as follows:

$$
p\left(\hat{\mathbf{d}}^{(k)} \mid \sigma_{\varepsilon}, \mathscr{M}_{j}^{(k)}\right)=\prod_{\ell=1}^{N_{m}} p\left(\hat{d}_{\ell}{ }^{(k)} \mid \sigma_{\varepsilon}, \mathscr{M}_{j}^{(k)}\right)
$$




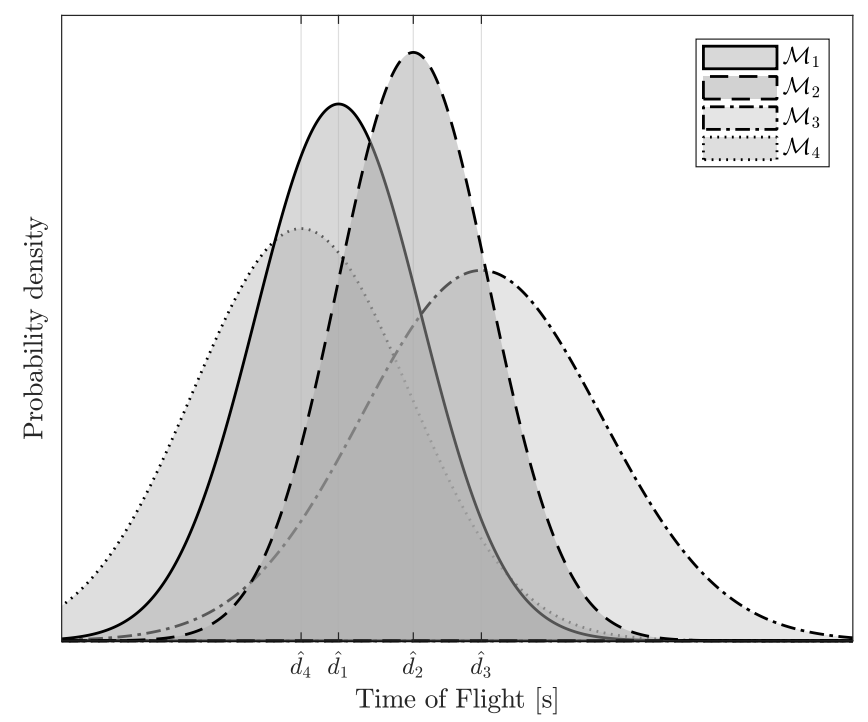

Figure 2: Likelihood functions derived from each time-of-flight $\left(\hat{d}_{j}{ }^{(k)}\right)$. The standard deviation provided by level I of the proposed model ranking is expected to have different values in each model-class. The time-of-flight data are then substituted in the likelihood function $p\left(\hat{\mathbf{d}}^{(k)} \mid \sigma_{\varepsilon}, \mathscr{M}_{j}^{(k)}\right)$.

Therefore, Equation (7) rewrites as:

$$
p\left(\sigma_{\varepsilon} \mid \hat{\mathbf{d}}^{(k)}, \mathscr{M}_{j}^{(k)}\right) \propto\left\{\prod_{\ell=1}^{N_{m}} p\left(\hat{d}_{\ell}^{(k)} \mid \sigma_{\varepsilon}, \mathscr{M}_{j}^{(k)}\right)\right\} p\left(\sigma_{\varepsilon} \mid \mathscr{M}_{j}^{(k)}\right)
$$

Furthermore, it is observed that the evaluation of the normalizing constant $c$ in Equation (7) cannot usually be evaluated analytically except for special cases based upon linear models and Gaussian uncertainties [38]. However, stochastic simulation based on MCMC methods [39, 40] can be used to obtain samples from the posterior avoiding the evaluation of $c$, as shown in the next section.

\subsection{Model class assessment}

The probabilistic approach for model class assessment is motivated by the uncertainty about the TF model based on the assumed hypotheses and simplifications adopted for its formulation [32, 33]. Once the posterior $p\left(\sigma_{\varepsilon} \mid \hat{\mathbf{d}}^{(k)}, \mathscr{M}_{j}^{(k)}\right)$ is obtained, the plausibility of the model class $\mathscr{M}_{j}^{(k)}$ can be obtained by applying the Total Probability Theorem as:

$$
\begin{aligned}
P\left(\mathscr{M}_{j}^{(k)} \mid \hat{\mathbf{d}}^{(k)}\right) & =\int_{\Theta} P\left(\mathscr{M}_{j}^{(k)} \mid \hat{\mathbf{d}}^{(k)}, \sigma_{\varepsilon}\right) p\left(\sigma_{\varepsilon} \mid \hat{\mathbf{d}}^{(k)}\right) d \sigma_{\varepsilon}= \\
& =\int_{\Theta} \frac{p\left(\hat{\mathbf{d}}^{(k)} \mid \mathscr{M}_{j}^{(k)}, \sigma_{\varepsilon}\right) P\left(\mathscr{M}_{j}^{(k)} \mid \mathbf{M}\right)}{\sum_{\ell=1}^{N_{m}} p\left(\hat{\mathbf{d}}^{(k)} \mid \mathscr{M}_{\ell}^{(k)}, \sigma_{\varepsilon}\right) P\left(\mathscr{M}_{\ell}^{(k)} \mid \mathbf{M}\right)} p\left(\sigma_{\varepsilon} \mid \hat{\mathbf{d}}^{(k)}\right) d \sigma_{\varepsilon}
\end{aligned}
$$

where $p\left(\sigma_{\varepsilon} \mid \hat{\mathbf{d}}^{(k)}\right)$ denotes the posterior PDF obtained by Equation 10 . Equation (11) can be simplified by applying 
the asymptotic Laplace's approximation [32] as follows:

$$
P\left(\mathscr{M}_{j}^{(k)} \mid \hat{\mathbf{d}}^{(k)}\right) \approx \frac{p\left(\hat{\mathbf{d}}^{(k)} \mid \mathscr{M}_{j}^{(k)}, \sigma_{M_{j}}\right) P\left(\mathscr{M}_{j}^{(k)}\right)}{\sum_{\ell=1}^{N_{m}} p\left(\hat{\mathbf{d}}^{(k)} \mid \mathscr{M}_{\ell}^{(k)}, \sigma_{M_{j}}\right) P\left(\mathscr{M}_{\ell}^{(k)}\right)}
$$

where the conditioning on $\mathbf{M}$ has been suppressed for simplicity and $\sigma_{M_{j}}$ is the maximum a posteriori (MAP) value of the posterior PDF $p\left(\sigma_{\varepsilon} \mid \hat{\mathbf{d}}^{(k)}, \mathscr{M}_{j}^{(k)}\right)$, i.e.:

$$
\sigma_{M}=\underset{\sigma_{\varepsilon}}{\arg \max } p\left(\sigma_{\varepsilon} \mid \hat{\mathbf{d}}^{(k)}, \mathscr{M}_{j}^{(k)}\right)
$$

\subsection{Hyper-robust model estimation}

The probability-based ranking of the model classes $\mathscr{M}_{j}^{(k)}$ obtained above provides information about the degree of belief of the $j$-th TF model class for each sensor. However, a hyper-robust model [32] is proposed to account for the uncertainties held by all the model classes, thus providing a rigorous tool to address the model class selection. The hyper-robust model for a specific sensor $k$ is defined as a weighted average of each TF model as follows:

$$
p\left(\tilde{d}^{(k)} \mid \mathbf{M}\right)=\sum_{\ell=1}^{N_{m}} p\left(\tilde{d}^{(k)} \mid \mathscr{M}_{\ell}^{(k)}, \sigma_{M_{\ell}}\right) P\left(\mathscr{M}_{\ell}^{(k)} \mid \hat{\mathbf{d}}^{(k)}\right) \cong \mathscr{N}\left(\sum_{\ell=1}^{N_{m}} w_{\ell}^{(k)} \hat{d}_{\ell}^{(k)}, \sum_{\ell=1}^{N_{m}}\left(w_{\ell}^{(k)} \sigma_{M_{\ell}}\right)^{2}\right)
$$

where $\tilde{d}^{(k)}$ are the possible ToF values and $w_{\ell}^{(k)}$ are the weights, given by the posterior probabilities of the $\ell$-th model class $P\left(\mathscr{M}_{\ell}^{(k)} \mid \hat{\mathbf{d}}^{(k)}\right)$. Given that each stochastic model is assumed to be distributed as a Gaussian function, a simplified expression for the hyper-robust Gaussian distribution is also provided in Equation (14). However, to address the damage localization problem, the use of a stochastic model as the input data would require an intensively computational effort. Instead, the mean value of the hyper-robust model in the $k$-th sensor, denoted as $D^{(k)}$, is adopted.

\section{Bayesian damage localization}

\subsection{Probabilistic description of ToF model}

In this section, the damage localization is addressed by a model-based BIP using an ellipse-based ToF model [28], which has been extensively used for damage localization in guided-waves based SHM. For this problem, $N_{p}$ actuatorsensor paths are considered in a plate-like structure to excite and receive Lamb waves for damage localization by screening changes of their ToF. To this end, the ToF information of the scattered signals can be theoretically obtained as follows [41]:

$$
\operatorname{ToF}^{(a-s)}=\frac{\sqrt{\left(X_{d}-X_{a}\right)^{2}+\left(Y_{d}-Y_{a}\right)^{2}}}{V_{a-d}}+\frac{\sqrt{\left(X_{d}-X_{s}\right)^{2}+\left(Y_{d}-Y_{s}\right)^{2}}}{V_{d-s}}
$$


where $\left(X_{d}, Y_{d}\right)$ are the coordinates of the damage, $\left(X_{a}, Y_{a}\right)$ are the actuator transducer coordinates, $\left(X_{s}, Y_{s}\right)$ are the coordinates of one arbitrary sensor transducer, and $V_{a-d}$ and $V_{d-s}$ are the wave propagation velocities of the actuatordamage and damage-sensor paths respectively. These velocities are the same under the assumption of isotropic materials and a concentrated damage within a bounded region, i.e. $V=V_{a-d}=V_{d-s}$. Alternatively, under the consideration of orthotropic materials, such as composite structures, both velocity terms would be dependent on the angle of the actuator-damage and damage-sensor paths, $V_{a-d}\left(\alpha_{a}\right)$ and $V_{d-s}\left(\alpha_{s}\right)$ respectively [23].

To probabilistically describe the ToF model given by Equation (15), uncertainties coming from the data, material properties, and also from the model itself, need to be accounted for. To this end, a set of uncertain model parameters $\mathbf{m}=\left\{X_{d}, Y_{d}, V\right\}$ are considered in this problem to describe the uncertainty about the damage coordinates as well as the wave propagation velocity. The set $\mathbf{m}$ of model parameters is augmented with a model error term $e \in \mathbb{R}$, resulting in a set of model parameters defined as $\boldsymbol{\theta}=\left\{\mathbf{m}, \sigma_{e}\right\}=\left\{X_{d}, Y_{d}, V, \sigma_{e}\right\} \in \boldsymbol{\Theta}$, where $\sigma_{e}$ is the standard deviation of the error term $e$ and $\Theta$ is the model parameter space. This set of parameters is further updated through Bayes’ Theorem, as will be explained below. The referred model error term $e \in \mathbb{R}$ is considered to account for the non-existence of a theoretical ToF model that fully represent the reality, so that:

$$
\operatorname{ToF}_{\mathscr{D}}^{(a-s)}=\operatorname{ToF}_{M}^{(a-s)}(\mathbf{m})+e=\operatorname{ToF}_{M}^{(a-s)}(\boldsymbol{\theta})
$$

where subscripts $M$ and $\mathscr{D}$ from $\operatorname{ToF}_{M}^{(a-s)}$ and $\operatorname{ToF}_{\mathscr{D}}^{(a-s)}$ refer to modeled and measured ToF, respectively. Note in Equation (16) that $e$ provides the discrepancy between $\operatorname{ToF}_{M}^{(a-s)}$ and $\operatorname{ToF}_{\mathscr{D}}^{(a-s)}$ values. By the PMIE, this error term can be conservatively described as a zero-mean Gaussian distribution with covariance $\sigma_{e}$ as $\mathscr{N}\left(0, \sigma_{e}\right)$. Thus, a probabilistic description of the ToF model from Equation 16 can be obtained as:

$$
p\left(\operatorname{ToF}_{\mathscr{D}}^{(a-s)} \mid \operatorname{ToF}_{M}^{(a-s)}(\boldsymbol{\theta})\right)=\left(2 \pi \sigma_{e}^{2}\right)^{-\frac{1}{2}} \exp \left(-\frac{1}{2}\left(\frac{\operatorname{ToF}_{\mathscr{D}}^{(a-s)}-\operatorname{ToF}_{M}^{(a-s)}(\boldsymbol{\theta})}{\sigma_{e}}\right)^{2}\right)
$$

Observe that Equation (17) provides a measurement of the similarity of the modeled and measured ToF. Also, note that Equation (17) provides a likelihood function for the $\operatorname{ToF}_{\mathscr{D}}^{(a-s)}$ data under the $\operatorname{ToF}_{M}^{(a-s)}(\boldsymbol{\theta})$ model.

\subsection{Model parameter estimation}

Given the likelihood function in Equation (17), one can obtain the posterior PDF of the model parameters given the ToF data $\mathbf{D}=\left\{D^{(1)}, \ldots, D^{(N)}\right\}$, where $N$ is the total number of sensors by applying the well-known Bayes' Theorem as:

$$
p(\boldsymbol{\theta} \mid \mathbf{D})=\frac{p(\mathbf{D} \mid \boldsymbol{\theta}) p(\boldsymbol{\theta})}{p(\mathbf{D})}
$$

where $p(\boldsymbol{\theta})$ is the prior PDF of the model parameters, and $p(\mathbf{D} \mid \boldsymbol{\theta})$ is the likelihood function for the set of data $\mathbf{D}$. Given the stochastic independence of the measurements, the likelihood can be expressed as $p(\mathbf{D} \mid \boldsymbol{\theta})=\prod_{k=1}^{N} p\left(D^{(k)} \mid \boldsymbol{\theta}\right)$, where 


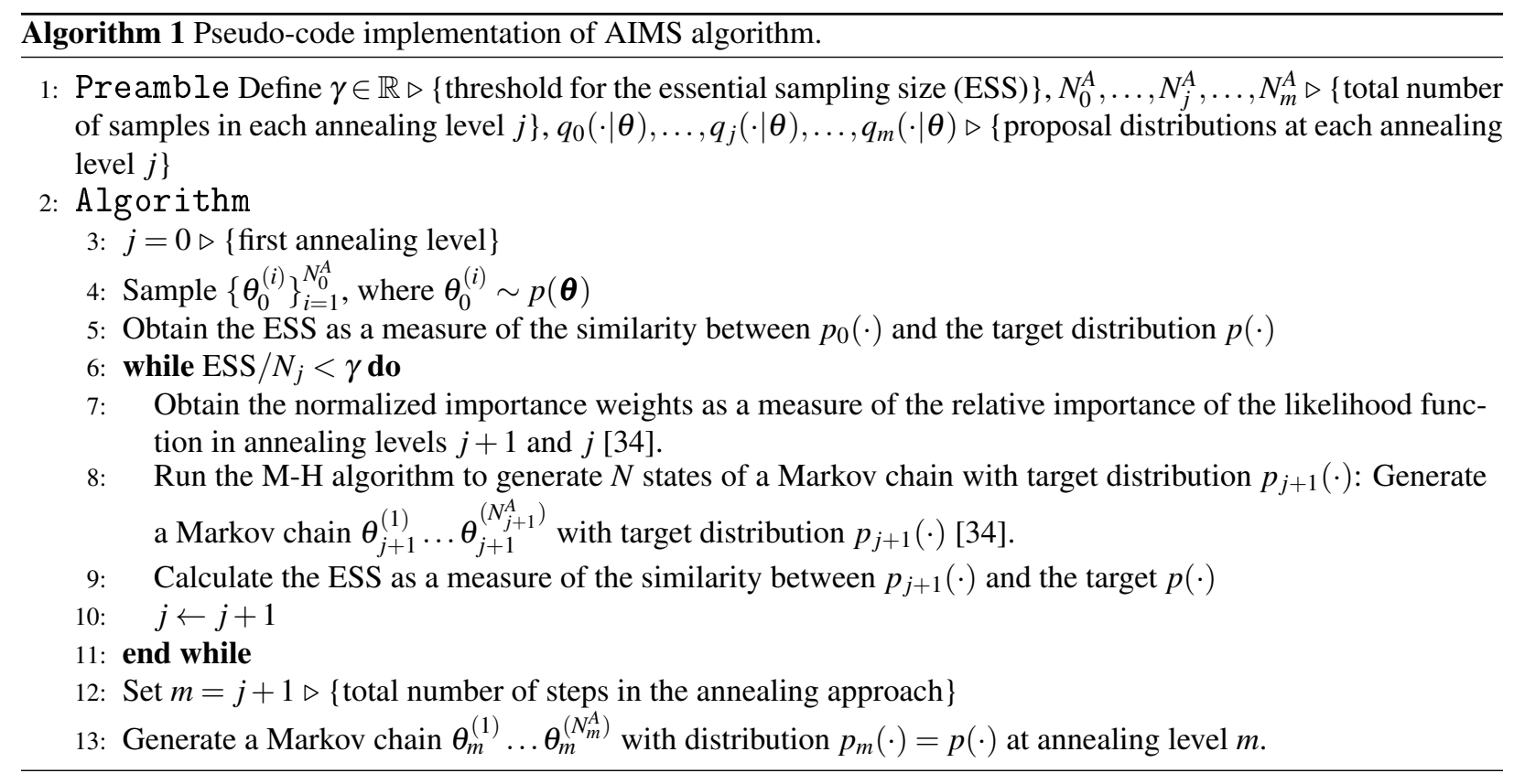

each factor $p\left(D^{(k)} \mid \boldsymbol{\theta}\right)$ is given by Equation $(17)$. Finally, $p(\mathbf{D})$ is the evidence of the data under the model specified by $\boldsymbol{\theta}$. This term, which acts as a normalizing factor within the Bayes' theorem, can be bypassed through sampling, e.g. using Markov Chain Monte Carlo (MCMC) methods [42]. Thus, Equation (18) can be rewritten as:

$$
p(\boldsymbol{\theta} \mid \mathbf{D}) \propto\left\{\prod_{k=1}^{N} p\left(D^{(k)} \mid \boldsymbol{\theta}\right)\right\} p(\boldsymbol{\theta})
$$

\subsection{Asymptotic independence Markov sampling algorithm}

In practice, the presence of multiple damage locations in plate-like structures is possible, thus the updating algorithm used to obtain the posterior PDF of the locations of such potential damage should be able to provide samples of a multimodal PDF. In the literature, the majority of available MCMC algorithms can identify multimodal posterior PDFs at the cost of increasing the computational burden, which can be exacerbated if large dimensional parameter spaces are explored, or by introducing ad-hoc algorithmic modifications [43]. To overcome this drawback, the asymptotic independence Markov sampling (AIMS) algorithm [34] is used here due to its efficiency to provide samples from multi-modal posterior PDFs. In AIMS algorithm, a posterior PDF $p(\cdot)$ is approximated using a combination of three well-known stochastic simulation methods. To this end, simulated annealing is used to obtain the target distribution $p(\cdot)$ from the prior distribution by sampling intermediate distributions $p_{j}(\cdot)$ through a random walk $M-H$. The intermediate distributions $p_{j}(\cdot)$ are approximated by using importance sampling. A pseudo-code implementation of AIMS method is provided as Algorithm 1 


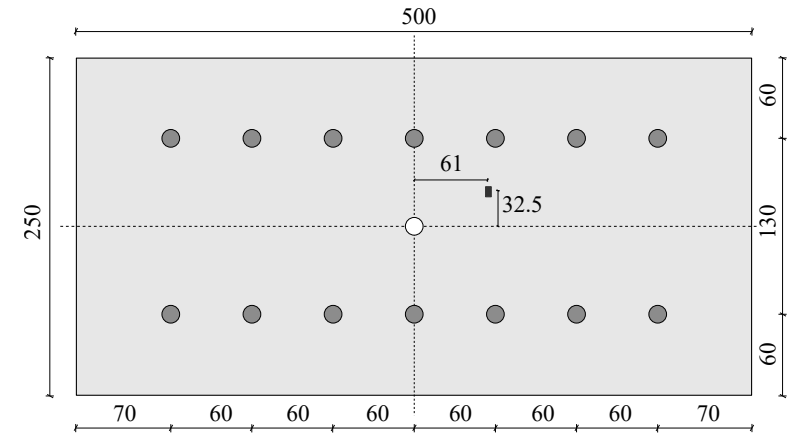

(a) Case study 1 considering one damage locations and 14 sensors.

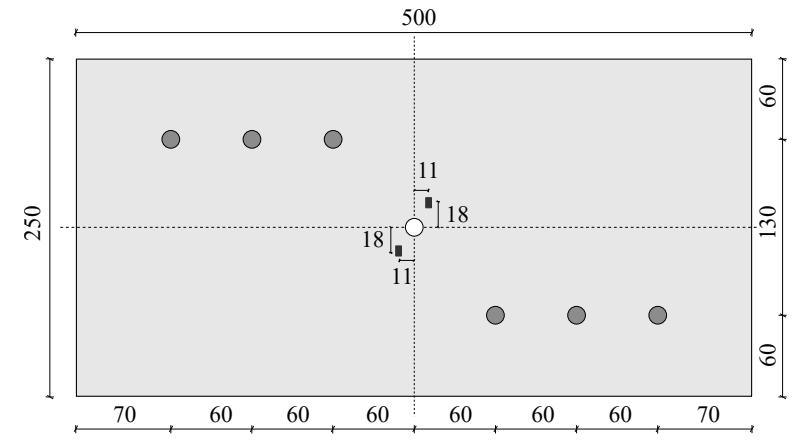

(b) Case study 2 considering two damage locations and 6 sensors.

Figure 3: Representation of the aluminum plates considered in both case studies along with the position and layout of the sensors (dimensions expressed in millimetres). The white circle represents the actuator, which is positioned in the center of the plate. Damage is represented using dark rectangles.

\section{Case studies}

In this section, two case studies are presented to validate the proposed model class selection methodology using a set of guided-waves synthetically generated by finite element modeling (FEM). The methodology is applied to two cases of damage detection and localization considering one and two damaged areas, respectively.

\subsection{Synthetic signal generation}

To numerically generate the input signals, Lamb waves are modeled using Abaqus® for the simulations. The waves are generated over a thin plate made of aluminum-based alloy 2024-T351 with dimensions $0.5 \mathrm{~m} \times 0.25 \mathrm{~m}$, as depicted in Figure 3 (see further properties about the aluminum alloy 2024-T351 in Table 1). In Figure 3)(panel (a)), sensor numbering is established starting from S1 for the left-most upper sensor to S14 for the right-most down sensor. In panel (b), which corresponds to the case of two damage locations, sensors are analogously arranged starting from S1 to S6. For the ToF calculations, the Abaqus/Explicit module is used in this work for its effectiveness in simulating the transient behavior of the ultrasonic guided-waves.

Table 1: Material and structural properties (aluminum alloy 2024-T351 [44]) used in the Abaqus model.

\begin{tabular}{cccc}
\hline $\begin{array}{c}\text { Young's modulus } \\
{[\mathrm{GPa}]}\end{array}$ & $\begin{array}{c}\text { Poisson's ratio } \\
{[-]}\end{array}$ & $\begin{array}{c}\text { Density } \\
{\left[\mathrm{kg} / \mathrm{m}^{3}\right]}\end{array}$ & $\begin{array}{c}\text { Thickness } \\
{[\mathrm{mm}]}\end{array}$ \\
\hline 73.1 & 0.33 & 2780 & 1.5 \\
\hline
\end{tabular}

A 4-node, quadrilateral, stress-displacement shell element with reduced integration and a large-strain formulation, referred to as S4R element [45], is used for the plate model, which is uniformly meshed using square elements of $1 \mathrm{~mm}$ size. The element size is determined by the smallest wavelength $\lambda_{\min }$ of the guided-wave mode represented. A minimum of 10 nodes per wavelength is normally required to ensure the avoidance of spatial aliasing [46]. The signal excitation is modeled as a perpendicular point force generated as a sine tone-burst of 5 cycles centered at 


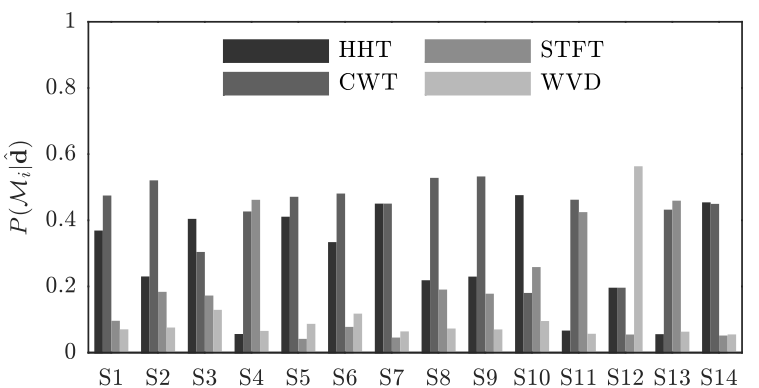

(a) $P\left(\mathscr{M}_{i} \mid \hat{\mathbf{d}}\right)$ for case study 1

\subsection{Model selection results} Section 3.3

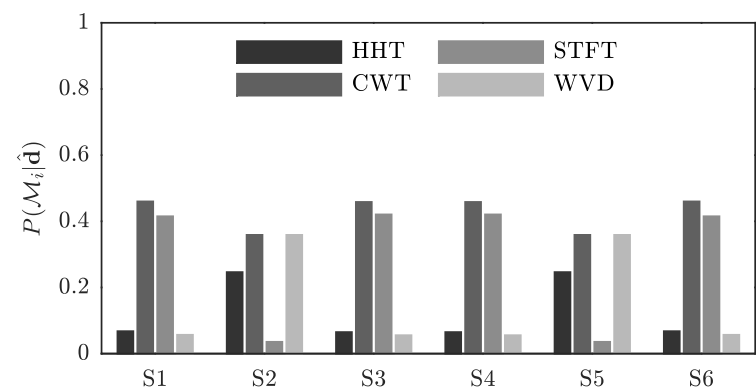

(b) $P\left(\mathscr{M}_{i} \mid \hat{\mathbf{d}}\right)$ for case study 2

Figure 4: Posterior probability $P\left(\mathscr{M}_{i} \mid \hat{\mathbf{d}}\right)$.

a frequency $f=100 \mathrm{kHz}$. This frequency is selected to avoid extra complexity in the signal post-processing due to the appearance of possible higher order guided-waves modes. When the frequency is maintained at relatively low values, only both anti-symmetric 0 (A0) and symmetric 0 (S0) modes are excited [46]. Given that the wave propagation velocity of the mode ${ }^{1}$ captured by the model is around $V=1950 \mathrm{~m} / \mathrm{s}$, the maximum element size would be $\lambda / 10=(V / f) / 10=1.95 \mathrm{~mm}$. However, note that the selected element size $(1 \mathrm{~mm})$ is nearly half of the maximum value. Next, the damage is modeled as a rectangular hole of dimension $2 \mathrm{~mm} \times 4 \mathrm{~mm}$ for both case studies considered in this paper. Free boundary conditions are considered in both cases. The ultrasonic signals are then received by the sensors in both undamaged and damaged cases. Afterwards, signals from both states are subtracted, thus the scattered information from the damage is obtained, as described in Section 4.1

As previously mentioned, the simulated response of the plate to Lamb waves is used as data within the Bayesian framework. First, the standard deviation parameter $\sigma_{\varepsilon}$ is defined as $\sigma_{\varepsilon}=\rho \cdot \hat{d}_{j}^{(k)}$, where $\hat{d}_{j}^{(k)}$ is the time of arrival at the $k$-th sensor using the $j$-th TF model, and $\rho$ is a factor defined within a sufficiently large interval, which in this example is taken as $(0,0.5]$. Therefore, the prior PDF of $\sigma_{\varepsilon}$ can be expressed as a uniform distribution over the referred interval, i.e. $p\left(\sigma_{\varepsilon}\right)=\mathscr{U}\left(0,0.5 \cdot \hat{d}_{j}^{(k)}\right)$. The posterior PDF $p\left(\sigma_{\varepsilon} \mid \hat{\mathbf{d}}^{(k)}, \mathscr{M}_{j}^{(k)}\right)$ is obtained through samples using the Metropolis-Hasting (M-H) algorithm (see a pseudo-code implementation in Appendix A with $T_{S}=40000$ and a Gaussian proposal distribution, i.e., $q\left(\theta^{\prime} \mid \theta\right)=\mathscr{N}(\theta, \sigma)$, where $\sigma$ is the standard deviation of the M-H random walk which is selected such that the acceptance rate $r$ lies within the interval [0.2,0.4] [47.-49]. The maximum a posteriori (MAP) parameter is then computed and introduced as an input for the model class selection problem, as explained in

The resulting posterior probabilities from Equation (12) are subsequently used to rank the candidate TF models for each of the sensors, as shown in Figures $4 a$ and $4 b$ for case study 1 and 2, respectively. Observe from these results

\footnotetext{
${ }^{1}$ The referred mode is the anti-symmetric mode (A0) since only the bending mode can be captured with shell elements
} 
Table 2: Time of arrival $\left(D^{(k)}\right)$ obtained as the mean of the hyper-robust models given by Equation 14 .

\begin{tabular}{|c|c|c|c|c|}
\hline & Sensor & $\begin{array}{c}\text { Time } \\
D^{(k)}[\mu \mathrm{s}]\end{array}$ & Sensor & $\begin{array}{c}\text { Time } \\
D^{(k)}[\mu \mathrm{s}]\end{array}$ \\
\hline \multirow{7}{*}{ 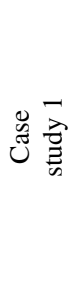 } & $\mathrm{S} 1$ & 157.53 & S8 & 163.33 \\
\hline & $\mathrm{S} 2$ & 125.29 & S9 & 136.23 \\
\hline & S3 & 98.125 & $\mathrm{~S} 10$ & 114.66 \\
\hline & S4 & 71.539 & S11 & 94.115 \\
\hline & S5 & 49.164 & $\mathrm{~S} 12$ & 83.664 \\
\hline & S6 & 70.242 & S13 & 96.235 \\
\hline & S7 & 98.500 & S14 & 116.35 \\
\hline \multirow{3}{*}{ 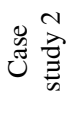 } & $\mathrm{S} 1$ & 109.96 & S4 & 54.577 \\
\hline & $\mathrm{S} 2$ & 81.214 & S5 & 81.214 \\
\hline & S3 & 54.577 & S6 & 109.96 \\
\hline
\end{tabular}

that there is not a particularly predominating TF model for all the sensors. Nonetheless, the CWT model emerges as the most plausible one for a considerable majority of sensors, i.e., 8 out of 14 sensors for the case study 1 , and 4 out of 6 , for case study 2 . Therefore, if a single TF model had to be selected for damage identification, a rational selection based on these results would be to choose the CWT model, since the better representation of the given data for the majority of sensors is provided by this choice. This output is in agreement with most of the authors in the literature who select CWT model to obtain the ToF from the scattered signals [23, 24]. Notwithstanding, a hyper-robust model can be obtained by applying Equation (14) using the posterior probabilities of each model class. The ToFs in this case are obtained by a model average from the probabilistic model from each sensor, as shown in Table 2. These values are subsequently used to reconstruct the damage in the BIP of damage localization, which is shown next.

\subsection{Damage localization and reconstruction}

Once the TF models are ranked and the hyper-robust TF model is obtained, the mean values of each hyper-robust model for each sensor are used as ToF data $\mathbf{D}$ within the BIP of damage localization, described in Section 4 The prior information of the model parameters has been defined as a uniform distribution for the damage position and dispersion parameter $\left(X \sim \mathscr{U}(-0.25,0.25) \mathrm{m}, Y \sim \mathscr{U}(-0.125,0.125) \mathrm{m}\right.$, and $\left.\sigma_{e} \sim \mathscr{U}\left(0,10^{-4}\right)\right)$, and a Gaussian PDF for the velocity $V \sim \mathscr{N}\left(v, \sigma_{v}\right)$, where $v=1950 \mathrm{~m} / \mathrm{s}$ and $\sigma_{v}=40 \mathrm{~m} / \mathrm{s}$. The posterior PDF of model parameters $\boldsymbol{\theta}$ is obtained in this case using the AIMS algorithm, with a threshold value $\gamma=1 / 2,10^{5}$ samples per annealing level, and a Gaussian PDF as proposal distribution, i.e. $q\left(\theta^{\prime} \mid \theta\right)=\mathscr{N}(\theta, \sigma)$, where $\sigma$ is the standard deviation of the M-H random walk which is again selected such that the acceptance rate $r$ lies within the interval [0.2,0.4]. Figure 5 shows the inferred damage position for the aluminum plates of case study 1 and 2, respectively, using the hyper-robust TF model obtained by Equation (14). The hyper-robust model is obtained for each sensor by model averaging weighted using the posterior plausibilities of the TF models, showed in Figure 4 It is observed that the damage position is efficiently reconstructed with the BIP methodology presented in this paper. The results also show that for the particular case study 2, the multi-modality due to dual damage position is well addressed using the AIMS algorithm. 


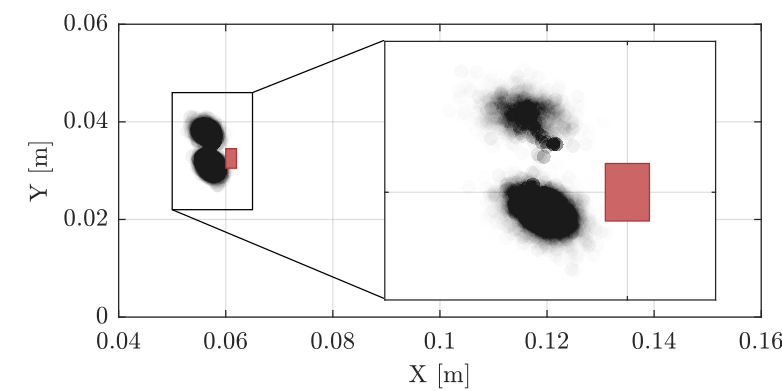

(a) Estimated and actual damage position $(X-Y)$ for the case study 1

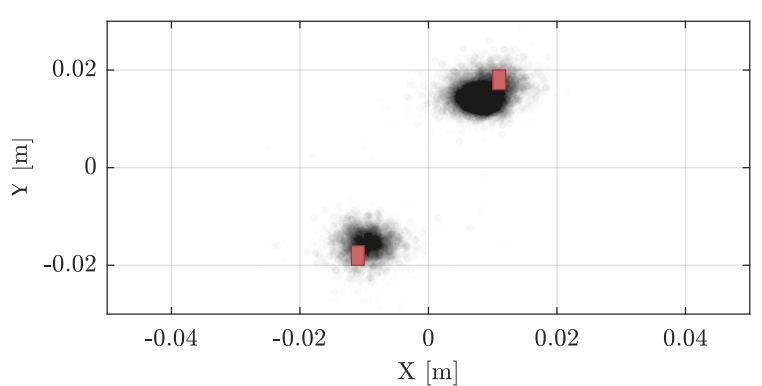

(b) Estimated and actual damage position $(X-Y)$ for the case study 2

Figure 5: Damage location estimation for the plate of case study 1 and 2. The actual position of the center of the damage is depicted using red rectangles which are represented in actual dimensions.

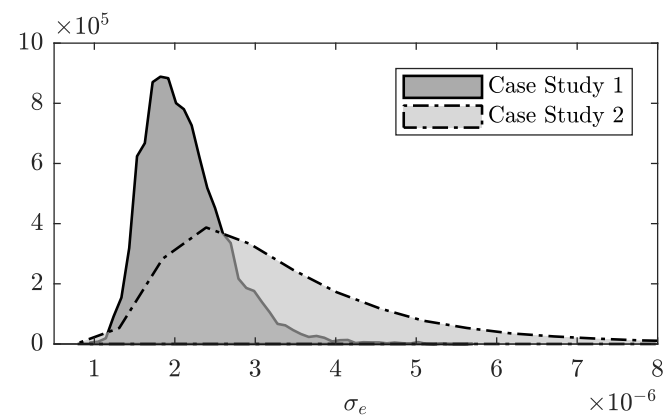

(a) Dispersion parameter $\left(\sigma_{e}\right)$.

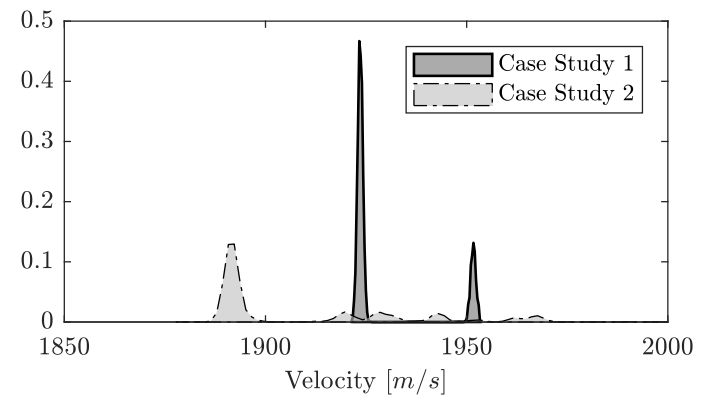

(b) Wave propagation velocity $(V)$.

Figure 6: Posterior PDFs of the $\sigma_{e}$ and $V$ parameters for both case study 1 and 2.

The marginal posterior distributions of the other two parameters used by the BIP of damage localization, namely the standard deviation factor of the likelihood function $\sigma_{e}$ and the wave propagation velocity $V$, are depicted in Figure 6 . In case study 1, a lower level of dispersion in both parameters, $\sigma_{e}$ and $V$, is observed, whereas in case study 2, a higher dispersion is obtained.

\section{Discussion}

The proposed Bayesian methodology for damage localization has been exemplified using two case studies presented in Section 5. For each of the sensors, a Bayesian model class assessment framework is proposed to rank the candidate TF models, according to relative plausibilities that measure the relative degree of belief of the candidate model class in interpreting the raw signal acquired by the sensor. These relative plausibilities are then used to obtain a hyper-robust TF model for each sensor, which provides a higher level of robustness to damage localization than just taking the most plausible TF model among the candidate set. This robustness is clearly manifested in Figure 5a, where 
damage position is identified in two plausible regions close to the actual damage position; an unjustified TF model choice would lead to a biased localization due to unconsidered model uncertainty. The same behavior can be also observed in Figure $6 \mathrm{~b}$ for the reconstruction of the wave propagation velocity parameter. Note that the ultrasonic data used in both case studies are synthetically obtained through FEM, although the methodology is entirely applicable to real ultrasonic signals. However, for real ultrasonic data, the uncertainty in the damage localization would be higher due to electronic noise or other measurement errors coming from, for instance, imperfect sensor bonding.

Then, a damage localization BIP using an ellipse-based model is applied to reconstruct the damage position using the AIMS algorithm as Bayesian updating algorithm. The data $\mathbf{D}$ are obtained by using the mean of the hyper-robust model, given by Equation (14) for each sensor independently. The damage location has been remarkably inferred in both case studies. However, a higher dispersion in the $X-Y$ parameters (larger localization uncertainty) has been found in case study 2 compared to case study 1, as can be observed in Figure 5 . In addition, a higher dispersion is identified in the marginal distribution of the standard deviation parameter $\sigma_{e}$ in case study 2 . This could be explained due to the nature of the likelihood function from Equation (17), which is a Gaussian distribution. In order to properly identify the two damage positions in this case study, the posterior values of $\sigma_{e}$ (which is an updatable parameter) need to increase, hence leading to a higher dispersion in the damage localization as well as in the velocity parameter reconstruction, as observed in Figure 6 This points out a limitation of the proposed methodology when dealing with multiple damage locations. In this context, a desirable further work would be the exploration of optimal likelihood functions to deal with damage multi-modality.

The robustness of the proposed methodology can then be observed by comparing the Bayesian identification results against those obtained using a deterministic approach. A well-known method to address inverse problems efficiently, but deterministically, is by the use of genetic algorithms (GA) [50]. GA's are used to find the value of the parameters that minimizes a cost function, which in this case can be defined as $\left\|\operatorname{ToF}_{\mathscr{D}}^{(a-s)}-\operatorname{ToF}_{M}^{(a-s)}(\mathbf{m})\right\|_{2}^{2}$, a $L_{2}$-norm of the residual time of flight between the model and the data. Figure 7 depicts the comparison between the damage identification results obtained using the proposed Bayesian methodology and a deterministic GA-based inverse problem. To better highlight the robustness of the proposed Bayesian damage identification methodology in the presence of uncertainties, a bias is artificially introduced in the velocity term as a $5 \%$ of deviation in the value obtained through Abaqus (from $1950 \mathrm{~m} / \mathrm{s}$ to $1850 \mathrm{~m} / \mathrm{s}$ ). Note that even with this small deviation, the damage localization results given by the GA become biased, whereas the results using Bayesian methodology are virtually immune to such variation. This simple example demonstrates the superiority of the proposed methodology in reconstructing the position of damage from guided-waves data, regardless of potential input errors.

Finally, it is worth mentioning that the position of the sensors plays a crucial role in the damage reconstruction. As observed in Figure 3, case studies 1 and 2 have different sensor selection and layout. In this paper, a former sensitivity study on the sensors positions was carried out to identify: (1) the best number and (2) locations of sensors for each case study. Therefore, given the influence of the aforementioned two factors in the reconstruction of the damage position, an immediate further work will be the exploration of an efficient methodology for optimal sensor 


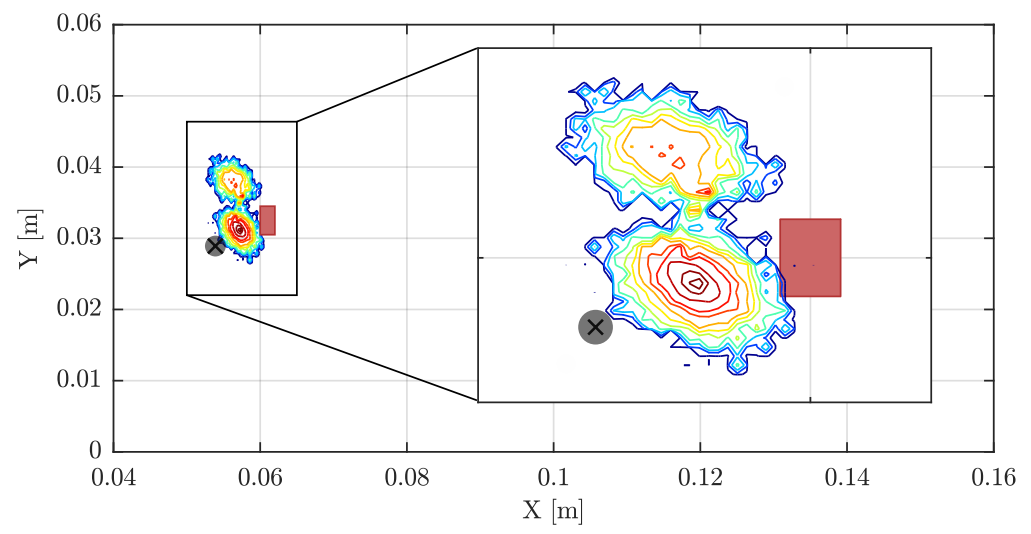

Figure 7: Comparison between a deterministic damage localization using GA (gray point) and the results obtained with the proposed Bayesian methodology.

configuration, based on rigorous probabilistic assumptions to deal with the aforementioned sources of uncertainties.

\section{Conclusions}

A Bayesian methodology for damage location using guided-waves is presented in this paper. This methodology allows accounting for several sources of uncertainty, like the epistemic uncertainty due to TF model selection, and the uncertainty coming from the measurement noise and variable material properties. The effectiveness of the method is shown through two case studies with one and two damaged areas, respectively. The following conclusions are drawn from this paper:

- The damage position can be accurately reconstructed using ToFs proving the effectiveness of the proposed multi-level Bayesian inverse problem methodology;

- The use of a hyper-robust TF model as an input for the damage localization Bayesian inverse problem leads to a more robust damage inference;

- The reconstruction of the two damage areas in case study 2 (multi-modality) has been remarkably addressed by using the AIMS algorithm. However, under this scenario of damage, an important increase in the posterior uncertainty of the model parameters is obtained.

Further research work is under consideration with regards to: (1) the assessment of a suitable likelihood function to efficiently deal with multi-modal damage scenarios, (2) devising a rigorous technique for optimal sensor configuration in ultrasonic guided-waves based SHM, and (3) the influence of different types of damage in the performance of the proposed methodology. 


\section{Acknowledgements}

This paper is part of the SAFE-FLY project that has received funding from the European Union's Horizon 2020 research and innovation programme under the Marie Sklodowska-Curie grant agreement No 721455.

\section{Appendix A. Metropolis-Hastings simulation for Bayesian updating}

The M-H algorithm generates samples from a specially constructed Markov chain whose stationary distribution is the required posterior PDF $p(\boldsymbol{\theta} \mid \hat{\mathbf{d}}, \mathscr{M})$. By sampling a candidate model parameter $\boldsymbol{\theta}^{\prime}$ from a proposal distribution $q\left(\boldsymbol{\theta}^{\prime} \mid \boldsymbol{\theta}^{\zeta}\right)$, the M-H obtains the state of the chain at $\zeta+1$, given the state at $\zeta$, specified by $\boldsymbol{\theta}^{\zeta}$. The candidate parameter $\boldsymbol{\theta}^{\prime}$ is accepted (i.e., $\boldsymbol{\theta}^{\zeta+1}=\boldsymbol{\theta}^{\prime}$ ) with probability $\min \{1, r\}$, and rejected (i.e., $\boldsymbol{\theta}^{\zeta+1}=\boldsymbol{\theta}^{\zeta}$ ) with the remaining probability $1-\min \{1, r\}$, where:

$$
r=\frac{p\left(\hat{\mathbf{d}} \mid \boldsymbol{\theta}^{\prime}, \mathscr{M}\right) p\left(\boldsymbol{\theta}^{\prime} \mid \mathscr{M}\right) q\left(\boldsymbol{\theta}^{\zeta-1} \mid \boldsymbol{\theta}^{\prime}\right)}{p\left(\hat{\mathbf{d}} \mid \boldsymbol{\theta}^{\zeta-1}, \mathscr{M}\right) p\left(\boldsymbol{\theta}^{\zeta-1} \mid \mathscr{M}\right) q\left(\boldsymbol{\theta}^{\prime} \mid \boldsymbol{\theta}^{\zeta-1}\right)}
$$

The process is repeated until $T_{S}$ samples have been generated so that the monitored acceptance rate (ratio between accepted M-H samples over total amount of samples) reaches an asymptotic behaviour. A pseudo-code description of this method is provided below as Algorithm 2

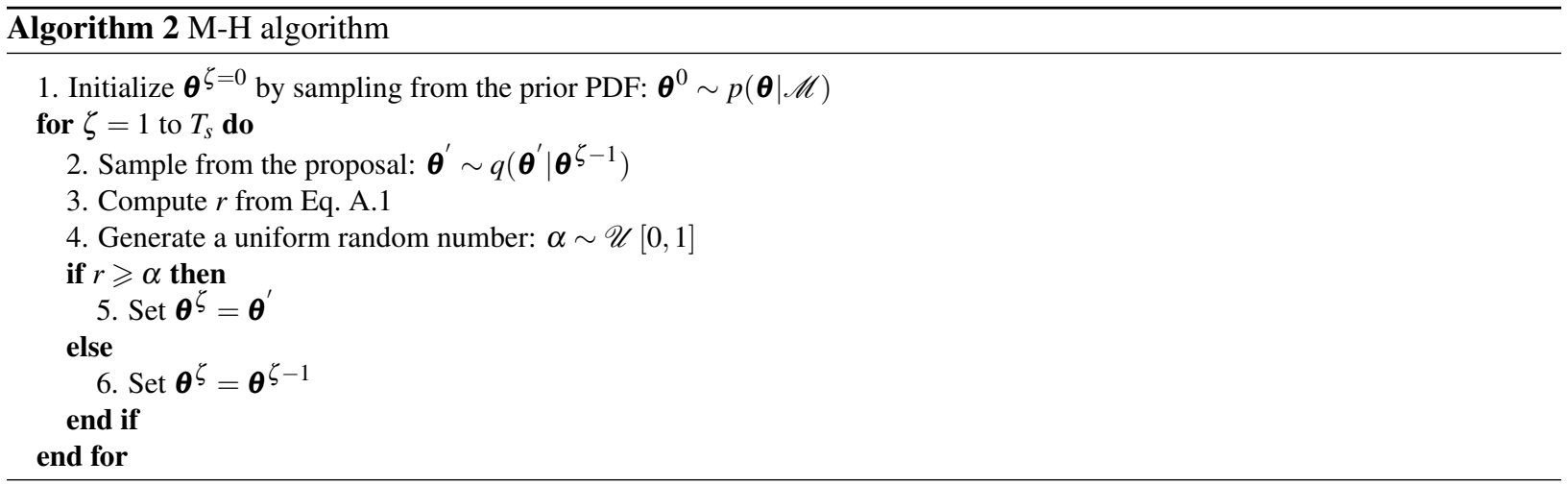

\section{References}

[1] J. Achenbach, Wave Propagation in Elastic Solids, North-Holland Publishing Company/American Elsevier, 1973.

[2] Z. Su, L. Ye, Y. Lu, Guided Lamb waves for identification of damage in composite structures: A review, Journal of sound and vibration $295(3-5)(2006) 753-780$.

[3] G. Aranguren, P. Monje, V. Cokonaj, E. Barrera, M. Ruiz, Ultrasonic wave-based structural health monitoring embedded instrument, Review of Scientific Instruments 84 (12) (2013) 125106

[4] P. M. Monje, L. Casado, G. Aranguren, V. Cokonaj, E. Barrera, M. Ruiz, Integrated electronic system for ultrasonic structural health monitoring, in: European workshop on structural health monitoring, 2012, pp. 1-8.

[5] A. Alcaide, E. Barrera, M. Ruiz, G. Aranguren, Damage detection on Aerospace structures using PAMELA SHM System, in: 6th International Symposium on NDT in Aerospace, Madrid, 2014. 
[6] V. Giurgiutiu, Chapter 9 - Impact and Acoustic Emission Monitoring for Aerospace Composites SHM, in: V. Giurgiutiu (Ed.), Structural Health Monitoring of Aerospace Composites, Academic Press, Oxford, 2016, pp. 317 - 394.

[7] A. Tobias, Acoustic-emission source location in two dimensions by an array of three sensors, Non-destructive testing 9 (1) (1976) 9-12.

[8] J. Park, F.-K. Chang, System identification method for monitoring impact events, in: Smart Structures and Materials 2005: Smart Sensor Technology and Measurement Systems, Vol. 5758, International Society for Optics and Photonics, 2005, pp. 189-201.

[9] J. F. Markmiller, F.-K. Chang, Sensor network optimization for a passive sensing impact detection technique, Structural Health Monitoring 9 (1) (2010) 25-39.

[10] L. E. Mujica, J. Vehí, W. Staszewski, K. Worden, Impact damage detection in aircraft composites using knowledge-based reasoning, Structural Health Monitoring 7 (3) (2008) 215-230.

[11] C. Hiche, C. K. Coelho, A. Chattopadhyay, A strain amplitude-based algorithm for impact localization on composite laminates, Journal of Intelligent Material Systems and Structures 22 (17) (2011) 2061-2067.

[12] H. Fu, C.-M. Vong, P.-K. Wong, Z. Yang, Fast detection of impact location using kernel extreme learning machine, Neural Computing and Applications 27 (1) (2016) 121-130.

[13] M. Mitra, S. Gopalakrishnan, Guided wave based structural health monitoring: A review, Smart Materials and Structures 25 (5) (2016) 053001 .

[14] L. Wang, F. Yuan, Active damage localization technique based on energy propagation of Lamb waves, Smart Structures and Systems 3 (2) (2007) 201-217.

[15] J. Chiachío, N. Bochud, M. Chiachío, S. Cantero, G. Rus, A multilevel Bayesian method for ultrasound-based damage identification in composite laminates, Mechanical Systems and Signal Processing 88 (2017) 462-477.

[16] C. H. Wang, J. T. Rose, F.-K. Chang, A synthetic time-reversal imaging method for structural health monitoring, Smart materials and structures 13 (2) (2004) 415.

[17] J. E. Michaels, T. E. Michaels, Guided wave signal processing and image fusion for in situ damage localization in plates, Wave motion 44 (6) (2007) 482-492.

[18] J. E. Michaels, A. J. Croxford, P. D. Wilcox, Imaging algorithms for locating damage via in situ ultrasonic sensors, in: Sensors Applications Symposium, 2008. SAS 2008. IEEE, IEEE, 2008, pp. 63-67.

[19] L. Cohen, Time-frequency Analysis: Theory and Applications, Prentice-Hall, Inc., Upper Saddle River, NJ, USA, 1995.

[20] N. E. Huang, Z. Shen, S. R. Long, M. C. Wu, H. H. Shih, Q. Zheng, N.-C. Yen, C. C. Tung, H. H. Liu, The empirical mode decomposition and the Hilbert spectrum for nonlinear and non-stationary time series analysis, in: Proceedings of the Royal Society of London A: mathematical, physical and engineering sciences, Vol. 454, The Royal Society, 1998, pp. 903-995.

[21] C. K. Chui, An Introduction to Wavelets, Academic Press Professional, Inc., San Diego, CA, USA, 1992.

[22] C. Bao, H. Hao, Z.-X. Li, X. Zhu, Time-varying system identification using a newly improved HHT algorithm, Computers \& Structures $87(23-24)$ (2009) 1611-1623.

[23] C. Fendzi, N. Mechbal, M. Rebillat, M. Guskov, G. Coffignal, A general Bayesian framework for ellipse-based and hyperbola-based damage localization in anisotropic composite plates, Journal of Intelligent Material Systems and Structures 27 (3) (2016) 350-374.

[24] G. Yan, A Bayesian approach for damage localization in plate-like structures using Lamb waves, Smart Materials and Structures 22 (3) (2013) 035012.

[25] M. S. Salmanpour, Z. Sharif Khodaei, M. Aliabadi, Impact damage localisation with piezoelectric sensors under operational and environmental conditions, Sensors 17 (5) (2017) 1178.

[26] B. Xu, L. Yu, V. Giurgiutiu, Advanced methods for time-of-flight estimation with application to lamb wave structural health monitoring, in: Proc. International Workshop on SHM, 2009, pp. 1202-1209.

[27] L. Peralta, X. Cai, P. Laugier, Q. Grimal, A critical assessment of the in-vitro measurement of cortical bone stiffness with ultrasound, Ultrasonics 80 (2017) 119-126.

[28] E. B. Flynn, M. D. Todd, P. D. Wilcox, B. W. Drinkwater, A. J. Croxford, Maximum-likelihood estimation of damage location in guided-wave 
structural health monitoring, in: Proceedings of the Royal Society of London A: Mathematical, Physical and Engineering Sciences, Vol. 467, The Royal Society, 2011, pp. 2575-2596.

[29] L. Yu, Z. Su, Application of kernel density estimation in Lamb wave-based damage detection, Mathematical problems in engineering 2012.

[30] E. D. Niri, S. Salamone, A probabilistic framework for acoustic emission source localization in plate-like structures, Smart Materials and Structures 21 (3) (2012) 035009.

[31] G. Rus, J. Chiachío, M. Chiachío, Logical inference for inverse problems, Inverse Problems in Science and Engineering 24 (3) (2016) $448-464$.

[32] J. L. Beck, Bayesian system identification based on probability logic, Structural Control and Health Monitoring 17 (7) (2010) $825-847$

[33] E. T. Jaynes, Information theory and statistical mechanics, Physical review 106 (4) (1957) 620.

[34] J. L. Beck, K. M. Zuev, Asymptotically independent Markov sampling: a new Markov chain Monte Carlo scheme for Bayesian inference, International Journal for Uncertainty Quantification 3 (5) (2013).

[35] K. M. Zuev, J. L. Beck, Global optimization using the asymptotically independent Markov sampling method, Computers \& Structures 126 (2013) 107-119.

[36] H. Jeong, Y.-S. Jang, Wavelet analysis of plate wave propagation in composite laminates, Composite Structures 49 (4) (2000) $443-450$.

[37] M. Niethammer, L. J. Jacobs, J. Qu, J. Jarzynski, Time-frequency representations of Lamb waves, The Journal of the Acoustical Society of America 109 (5) (2001) 1841-1847.

[38] M. S. Arulampalam, S. Maskell, N. Gordon, T. Clapp, A tutorial on particle filters for online nonlinear/non-Gaussian Bayesian tracking, IEEE Transactions on signal processing 50 (2) (2002) 174-188.

[39] N. Metropolis, A. W. Rosenbluth, M. N. Rosenbluth, A. H. Teller, E. Teller, Equation of state calculations by fast computing machines, The journal of chemical physics 21 (6) (1953) 1087-1092.

[40] W. K. Hastings, Monte Carlo sampling methods using Markov chains and their applications, Biometrika 57 (1) (1970) $97-109$.

[41] J.-B. Ihn, F.-K. Chang, Pitch-catch active sensing methods in structural health monitoring for aircraft structures, Structural Health Monitoring 7 (1) (2008) 5-19.

[42] F. Liang, C. Liu, J. Chuanhai, Advanced Markov Chain Monte Carlo Methods, Wiley Online Library, 2010.

[43] J. Besag, P. J. Green, Spatial statistics and Bayesian computation, Journal of the Royal Statistical Society. Series B (Methodological) (1993) 25-37.

[44] T. Dursun, C. Soutis, Recent developments in advanced aircraft aluminium alloys, Materials \& Design (1980-2015) 56 (2014) 862-871.

[45] ABAQUS, Abaqus Documentation, Dassault Systèmes, Providence, RI, USA (2016).

[46] D. Alleyne, P. Cawley, A two-dimensional Fourier transform method for the measurement of propagating multimode signals, The Journal of the Acoustical Society of America 89 (3) (1991) 1159-1168.

[47] M. Chiachío, J. Chiachío, G. Rus, J. L. Beck, Predicting fatigue damage in composites: A Bayesian framework, Structural Safety 51 (2014) $57-68$.

[48] A. Gelman, G. Roberts, W. Gilks, Efficient Metropolis jumping rules, Bayesian Statistics 5 (1996) 599-608.

[49] G. Roberts, J. Rosenthal, Optimal scaling for various Metropolis-Hastings algorithms, Statistical Science 16 (4) (2001) $351-367$.

[50] M. Gen, R. Cheng, Genetic algorithms and engineering optimization, Vol. 7, John Wiley \& Sons, 2000. 